\title{
The role of preoperative oral pregabalin on acute post-operative pain after orthopedic lower limb surgery
}

\author{
Girish Sahni ${ }^{1}$, Ayush Kumar Jain ${ }^{2 *}$, Sanjeev Sreen ${ }^{3}$, Sanjeev Kumar ${ }^{4}$, Arvind Kumar ${ }^{5}$ \\ ${ }^{1,3}$ Associate Professor, ${ }^{2,4}$ Senior Resident, ${ }^{5}$ Assistant Professor, Dept. of Orthopaedics, Government Medical College \& Rajindra Hospital, \\ Patiala, Punjab, India
}

*Corresponding Author: Ayush Kumar Jain

Email: ayush76@gmail.com

\begin{abstract}
Introduction: Postoperative pain after orthopedic surgery has remained a challenging problem, which prolongs hospital stay and early rehabilitation. Pregabalin comes under class of gabapentinoids that have been used in postoperative pain in arthroplasty and spine surgeries but studies regarding its role as preemptive analgesia in orthopedic limb surgeries are very few.

Aim: To study the efficacy and safety of pregabalin as preemptive medication for acute postoperative pain management in patients undergoing orthopedic lower limb fracture surgeries.

Materials and Methods: A randomized double blinded prospective study was undertaken. Sixty patients were enrolled with age between 18-70 years and were divided into 2 groups. Group A - received 150mg of oral pregabalin capsule, Group B - received matched colour empty capsules. Standard spinal anaesthesia was given. Breakthrough analgesia was given with Inj. Diclofenac $1.5 \mathrm{mg} / \mathrm{kg} \mathrm{i} / \mathrm{m}$. Assessment of pain was done with visual analog scale (VAS).

Results: There was marked reduction in postoperative VAS score in group A and the amount of breakthrough analgesia needed in the 24hour postoperative period was also much lower in the Group A as compared to the Group B.

Conclusion: 150mg of oral pregabalin as preemptive medication offers satisfactory postoperative analgesia in orthopaedic lower limb fracture surgeries with very few undesirable side effects.
\end{abstract}

Keywords: Postoperative pain, Pregabalin, Gabapentinoids, Preemptive analgesia, Diclofenac, Visual analog scale.

\section{Introduction}

Proper management of postoperative pain after orthopaedic surgery is a major challenge for the surgeon as it has great impact on the functional outcome. Postoperative pain is associated with many undesirable side effects like anxiety, tachycardia, increased hospital stay, patient discomfort and prolong rehabilitation. Principles of pain management include assessment of the severity of pain, meticulous use of analgesic drugs to anticipate the response to treatment to maximize the functional status, and quality of life. There are a number of analgesic drugs available in the market but none is able to provide desired outcome and each of them come with their fair share of side effects. Currently the technique of multimodal analgesia, in which a combination of drugs acting via different mechanism of action for pain relief, and preemptive analgesia, in which drugs are administered prior to surgery to prevent postoperative pain has gained significant popularity and have found to help in the alleviation of pain after surgery. ${ }^{1,2}$ This includes drugs such as local and regional anesthetic drugs, opiods, patientcontrolled analgesia (PCA), NSAIDs, centrally acting drugs and gabapentinoids, clonidine, and dexmedetomidine. ${ }^{3}$

GABA analogues such as pregabalin and gabapentin are the most common gabapentinoids being used. Role of pregabalin have been studied in arthroplasty and spine surgeries but not many studies regarding its role as preemptive analgesia in orthopedic limb fracture surgeries are available. ${ }^{4-6}$
Aim

The aim of the study is to compare the efficacy and safety of preoperative pregabalin in a dose of $150 \mathrm{mg}$ with a placebo on acute postoperative pain in patients scheduled for orthopaedic lower limb fracture surgeries under spinal anaesthesia

\section{Materials and Methods}

The current study was conducted in the Orthopaedics department, Govt. Medical College and Rajindra Hospital, Patiala, for duration of six months during January 2019 to June 2019. After approval from the local ethical committee, written informed consents were obtained from the patients who were planned to undergo surgeries for lower limb fractures under spinal anaesthesia. A randomized double blinded prospective study was undertaken. Sixty patients aged between 18-70 years were enrolled in the study and were randomly divided into 2 groups of 30 patients each. Exclusion criteria included known allergy to gabapentin or pregabalin, hepatic failure, renal failure, diabetes mellitus, psychiatric disorders, chronic gabapentin or pregabalin use, chronic pain and history of drug abuse.

Approximately $2 \mathrm{~h}$ prior to induction of anesthesia the oral medication was administered. Group A - received $150 \mathrm{mg}$ of oral pregabalin capsule $2 \mathrm{~h}$ prior to surgery, Group B - received matched colour placebo (capsules of similar appearance, but lacking the active ingredient of the drug). No other premedication was permitted. The patients were induced by standardized spinal anesthesia technique with $12-15 \mathrm{mg}$ of Bupivacaine $0.5 \%$ injected into the L4-L5 disc space by using 25 -gauge needle and the surgery was performed. 
The patients were assessed at 2, 4, 8, 16 and 24 hours after surgery for the severity of pain, the amount of breakthrough analgesia requirement and for any adverse effects like sedation, nausea \& vomiting, headache, dizziness or visual disturbance in the immediate postoperative period.

Assessment of acute postoperative pain was done by VAS. All patients were educated to convey the amount of pain they experienced using a 10-point Visual Analogue Scale (VAS), where 0 means no pain and 10 means extreme pain. As soon as VAS exceeded 4, patients were given Inj. Diclofenac $1.5 \mathrm{mg} / \mathrm{kg}$ intramuscular for breakthrough pain.

The Modified Ramsay sedation scale was used to assess the sedation; patients with a sedation scale of $>5$ were considered as sedated.

\section{Results}

The two study groups were similar with respect to demographic details such as age, sex and duration of surgery.

Average pain scores (VAS) were significantly lower in pregabalin group than placebo group at 2, 4, 8, 16 and 24 hours after surgery. VAS score in majority of the patients in the pregabalin group was $<4$. Only on nine occasions the score was $>4$ in Group A, whereas score $>4$ on sixteen occasions in Group B.

Table 1: Mean visual analogue score

\begin{tabular}{|l|c|c|c|}
\hline \multicolumn{1}{|c|}{ Variable } & Pregabalin & Placebo & P- Value \\
\hline Mean VAS score & & & \\
\hline 2h after surgery & $5.5 \pm 0.8$ & $7.3 \pm 1.1$ & $<0.001$ \\
\hline 4h after surgery & $6.4 \pm 1.2$ & $8.6 \pm 0.7$ & $<0.001$ \\
\hline 8h after surgery & $3.6 \pm 0.6$ & $7.8 \pm 0.5$ & $<0.0001$ \\
\hline 16h after surgery & $3.2 \pm 0.9$ & $5.4 \pm 0.8$ & $<0.001$ \\
\hline 24h after surgery & $2.4 \pm 1.2$ & $3.2 \pm 1.1$ & $<0.001$ \\
\hline
\end{tabular}

The average dose of Diclofenac required as post-operative breakthrough analgesia is also considerably lower in pregabalin group than placebo.

The sedations scores in terms of Modified Ramsay sedation score were more in the Group A as compared to group B. Sedation score $>5$ on 10 occasions in Group A and on 3 occasions in Group B.

Table 2: Modified Ramsey sedation score

\begin{tabular}{|l|c|c|c|c|c|}
\hline Variable & \multicolumn{2}{|c|}{ Pregabalin } & \multicolumn{2}{c|}{ Placebo } & $\begin{array}{c}\text { P- } \\
\text { Value }\end{array}$ \\
\hline SEDATION score & $<5$ & $>5$ & $<5$ & $>5$ & \\
\hline 2h after surgery & 28 & 2 & 30 & 0 & $<0.001$ \\
\hline 4h after surgery & 27 & 3 & 28 & 2 & $>0.001$ \\
\hline 8h after surgery & 25 & 5 & 29 & 1 & $<0.001$ \\
\hline 16h after surgery & 30 & 0 & 30 & 0 & $>0.001$ \\
\hline 24h after surgery & 30 & 0 & 30 & 0 & $>0.001$ \\
\hline
\end{tabular}

There were increased episodes of nausea at 2, $4 \mathrm{~h}$ after the surgery in group A but there was no statistical difference in terms of vomiting and dizziness between the groups.

\section{Discussion}

Acute pain has been a predictor of overall functional outcome after any major surgery, and inadequate management has been associated with poor patient satisfaction and may lead to development of chronic pain in many patients. Orthopaedic fractures are associated with severe pain and morbidity due to extensive bone and soft tissue damage and further exacerbation by surgery, thus adequate analgesia is required in the immediate postoperative period for proper pain relief and prevention of various adverse effects associated with it. Recent advances in molecular biology has led to development of better understanding of different pain pathways and the concept of multimodal and preemptive analgesia has come into play. ${ }^{2}$

Pregabalin is a cyclic GABA analogue which exerts a specific analgesic effect in neuropathic pain. It has been found to modulate a subset of neuronal voltage sensitive $\mathrm{Ca} 2+$ channels which contain $\alpha 2 \delta-1$ subunits. It is postulated that decreased entry of $\mathrm{Ca} 2+$ into the presynaptic neurons through these channels could reduce glutamate release, lowering neuronal excitability. ${ }^{7}$ Pregabalin is considered to be a first line drug for neuralgic pain due to diabetic neuropathy and postherpetic neuralgia. In addition it is also used in partial seizures, generalized anxiety disorders and chronic pain. Recently role of preemptive pregabalin for use in postoperative pain has been studied as it reduces over excitability of neurons in spinal cord and regulates the activity of excitatory neurotransmitters in brain. The usual dose used ranges from $50 \mathrm{mg}-600 \mathrm{mg}$ per day. The common side effects are mild sedation, tiredness, dizziness and unsteadiness. ${ }^{8,9}$

Current study showed a reduction in the postoperative VAS score during the $2,4,8,16$ and 24 hours period in the pregabalin group as compared to the placebo group. Akhavanakbari, in a study of sixty patients also produced similar results; they showed $150 \mathrm{mg}$ dose preoperative pregabalin is a reliable method in reducing pain and pethidine consumption in orthopaedic lower limb surgery. Similar results were reported by a number of authors in joint replacement, arthroscopic and laparoscopic surgeries. ${ }^{10-14}$ YaDeau and coworkers, in a study of 30 patients undergoing total knee arthroplasty, however reported that preoperative pregabalin has no beneficial effects. ${ }^{4}$

The dose of Pregabalin taken in this study was $150 \mathrm{mg}$ as previous studies with higher doses of pregabalin are associated with more side effects. A number of authors showed pregabalin $150 \mathrm{mg}$ is a better choice. ${ }^{14,15}$ Few studies used multiple doses of pregabalin pre and postoperatively and others showed only a single preoperative dose of pregabalin $150 \mathrm{mg}$ is sufficient. . $^{4,5}$

Headache, dizziness and sedation are the most commonly reported adverse effects of pregabalin (22$29 \%) .{ }^{16,17}$ Our study also concluded the same as sedation was the most common side effect followed by nausea. Similar results were reported by Sebastian B et $\mathrm{al}^{15}$ who found that hypotension $(33.33 \%)$ and dizziness $(26.66 \%)$ as the most common side effects. Akhavanakbari and colleagues however found that nausea and vomiting scores 
and sedation levels at $2 \mathrm{~h}$ and $6 \mathrm{~h}$ were reduced in the pregabalin group. ${ }^{14}$

\section{Limitation}

The study had limitation though as we used single preoperative dose of $150 \mathrm{mg}$ Pregabalin 2 hours before surgery. However, there is no consensus about the appropriate timing, dose and duration of the drug. Moreover, we conducted this study in a single hospital; therefore, the generalizability of our findings deserves further investigation in the future.

\section{Conclusion}

In conclusion, the current study demonstrated that single preoperative doses of pregabalin $150 \mathrm{mg}$ in patients undergoing orthopaedic lower limb fracture surgeries offers good postoperative analgesia and reduction in NSAIDs consumption in the $24 \mathrm{~h}$ postoperative period without any significant adverse effects.

\section{Source of funding}

None.

\section{Conflict of interest}

None.

\section{References}

1. Vadivelu N, Mitra S, Narayan D. Recent advances in postoperative pain management. Yale J Biol Med 2010;83:1125. Kissin I. Pre-emptive analgesia. Anaesthesiol 2000;93:1138-43.

2. Breivik H, Borchgrevink PC, Allen SM, Rosseland LA, Romundstad L. Assessment of pain. Br J Anaesth. 2008;101:17-24

3. Block BM, Liu SS, Rowlingson AJ, Cowan AR, Cowan JA, Jr, Wu CL. Efficacy of postoperative epidural analgesia: A metaanalysis. JAMA 2003;290:2455-63.

4. YaDeau JT, Lin Y, Mayman DJ, Goytizolo EA, Alexiades $\mathrm{MM}$ et al. Pregabalin and pain after total knee arthroplasty: a double-blind, randomized, placebo-controlled, multidose trial Br J Anaesth 2015;115(2):285-93.

5. Jain P, Jolly A, Bholla V, Adatia S, Sood J. Evaluation of efficacy of oral pregabalin in reducing postoperative pain in patients undergoing total knee arthroplasty. Indian J Orthop 2012;46:646-52.

6. Ozgencil E, Yalcin S, Tuna H, Yorukoglu D, Kecik Y. Perioperative administration of gabapentin 1,200 mg day-1 and pregabalin $300 \mathrm{mg}$ day-1 for pain following lumbar laminectomy and discectomy: A randomised, double-blinded, placebo-controlled study. Singapore Med J 2011;52:883-9.

7. Saraswat V, Arora V. Pre emptive use of gabapentin vs. pregabalin for acute postoperative pain after surgery under spinal anaesthesia. Indian J Anaesth 2008;52:829 34.

8. Gajraj NM. Pregabalin: Its Pharmacology and use in pain management. Anaesth Analg. 2007;105:1805-15.

9. Ghai A, Gupta M, Rana N, Wadhera R. The effect of pregabalin and gabapentin on preoperative anxiety and sedation: A double blind study. Anaesth Pain Intensive Care 2012;16:257-61.

10. Pandey CK, Priye S, Singh S, Singh U, Singh RB, Singh PK. Preemptive use of gabapentin significantly decreases postoperative pain and rescue analgesic requirements in laparoscopic cholecystectomy. Can J Anaesth 2004;51:358-63.
11. Jokela R, Ahonen J, Tallgren M, Haanpaa M, Korttila K. Premedication with pregabalin 75 or $150 \mathrm{mg}$ with ibuprofen to control pain after day-case gynaecological laparoscopic surgery. Br J Anaesth 2008;100:834-40.

12. Montazeri K, Kashefi P, Honarmand A. Pre-emptive gabapentin significantly reduces postoperative pain and morphine demand following lower extremity orthopaedic surgery. Singapore Med J 2007;48:748-51.

13. Bafna U, Rajarajeshwaran K, Khandelwal M, Verma AP. A comparison of effect of preemptive use of oral gabapentin and pregabalin for acute postoperative pain after surgery under spinal anaesthesia. J Anaesthesiol Clin Pharmacol 2014;30:373-7.

14. Akhavanakbari G, Entezarias1 M, Isazadehfar K, Mirzarahimi $\mathrm{T}$. The effects of oral pregabalin on postoperative pain of lower limb orthopedic surgery: A double blind, placebo controlled trial. Perspect Clin Res 2013;4:165-8.

15. Sebastian B, Talikoti AT, Nelamangala K, Krishnamurthy D. Effect of Oral Pregabalin as Preemptive Analgesic in Patients Undergoing Lower Limb Orthopedic Surgeries under Spinal Anaesthesia. J Clin Diagn Res 2016;10(7):UC01-4.

16. Rajendran I, Basavareddy A, Meher BR, Srinivasan S. Prospective, randomised, double blinded controlled trial of gabapentin and pregabalin as pre emptive analgesia in patients undergoing lower abdominal and limb surgery under spinal anaesthesia. Indian J Pain 2014;28:155-9.

17. Khetarpal R, Kataria AP, Bajaj S, Kaur H, Singh S. Gabapentin vs pregabalin as a premedication in lower limb orthopaedics surgery under combined spinal epidural technique. Anesth Essays Res 2016;10(2):262-7.

How to cite this article: Sahni G, Jain AK, Sreen S, Kumar S, Kumar A. The role of preoperative oral pregabalin on acute post-operative pain after orthopedic lower limb surgery. Int J Orthop Rheumatol 2019;5(2):557. 